\title{
Cirugía conservadora de urgencia en monorrena por rotura de angiomiolipoma múltiple
}

\author{
Aguilera Tubet C, Portillo Martín JA, Gutiérrez Baños JL, Ruiz Izquierdo F, Ballestero Diego R, \\ Zubillaga Guerrero S.
}

Servicio de Urología. Hospital Universitario Marqués de Valdecilla. Santander.

Actas Urol Esp. 2007;31(2):164-167

\section{RESUMEN}

CIRUGÍA CONSERVADORA DE URGENCIA EN MONORRENA POR ROTURA DE ANGIOMIOLIPOMA MÚLTIPLE

Se presenta el caso una hemorragia retroperitoneal debida a la rotura de un angiomiolipoma (AML) en una paciente monorrena. La paciente debuta con un shock hipovolémico por lo que es necesario realizar una cirugía de urgencia, pudiendo ser en este caso conservadora. Se revisan así mismo las distintas etiologías, diagnóstico y tratamiento de la hemorragia retroperitoneal espontánea.

Palabras clave: Hemorragia retroperitoneal. Angiomiolipoma múltiple. Cirugía conservadora.

\section{ABSTRACT}

EMERGENCY CONSERVATIVE SURGERY ON A UNIQUE KIDNEY WITH BROKEN MULTIPLE ANGIOMYOLIPOMA

A case of retroperitoneal hemorrhage due to the rupture of a multiple angiomyolipoma in a female with a unique kidney is described. Hipovolemic shock was the first symptom, being possible to perform on her a successful conservative surgery. Etiology, diagnostic methods and treatment of the spontaneous retroperitoneal hemorrhage are discussed.

Keywords: Retroperitoneal hemorrhage. Multiple angiomyolipoma. Conservative surgery.

$\mathrm{U}$ na de las principales causas de la hemorragia retroperitoneal espontánea son los tumores renales y entre ellos el angiomiolipoma (AML). En la mayoría de las ocasiones se puede llevar a cabo un tratamiento conservador con medidas de soporte y observación. En los casos en que se produzca una inestabilidad hemodinámica es mandatario una actitud agresiva, como primera elección la arteriografía con embolización del vaso sangrante y en segundo lugar la exploración quirúrgica. En los casos en que se hace necesaria la exploración quirúrgica, es difícil llevar a cabo una cirugía conservadora realizándose en muchas ocasiones la nefrectomía para el control de la hemorragia. Lo interesante del caso que comunicamos radica en lo mandatario de una cirugía conservadora, ya que la paciente era monorrena quirúrgica, ante una situación de extrema urgencia vital.

\section{CASO CLINICO}

Mujer de 48 años que acude al Servicio de Urgencias por un cuadro de dolor lumbar y abdominal, junto con decaimiento de 24 horas de evolución.

Como antecedentes personales destaca la realización de nefrectomía derecha de urgencia por hematoma retroperitoneal en 1983, en que la paciente presentó un cuadro similar al actual aunque más larvado. La anatomía patológica informó un riñón derecho con AML múltiples y hemorragia intensa capsular y perirrenal. Se investigó una 
posible esclerosis tuberosa lo cual fue descartada por el Servicio de Neurología. La paciente se pierde de seguimiento con posterioridad.

La paciente presentaba un abdomen patológico por lo que fue valorada en primer lugar por facultativos del Servicio de Cirugía General, quienes ante la sospecha clinica de una diverticulitis complicada, solicitan un TAC abdominal. El TAC abdominal se informó como nefrectomía derecha, AML múltiples en riñón izquierdo y gran hematoma retroperitoneal con extensión hasta la bifurcación de los vasos iliacos, con signos de sangrado activo, hemoperitoneo de aproximadamente 1 litro y dilatación de la pelvis renal izquierda (Fig. 1). Ante los hallazgos del TAC avisan al Servicio de Urología.

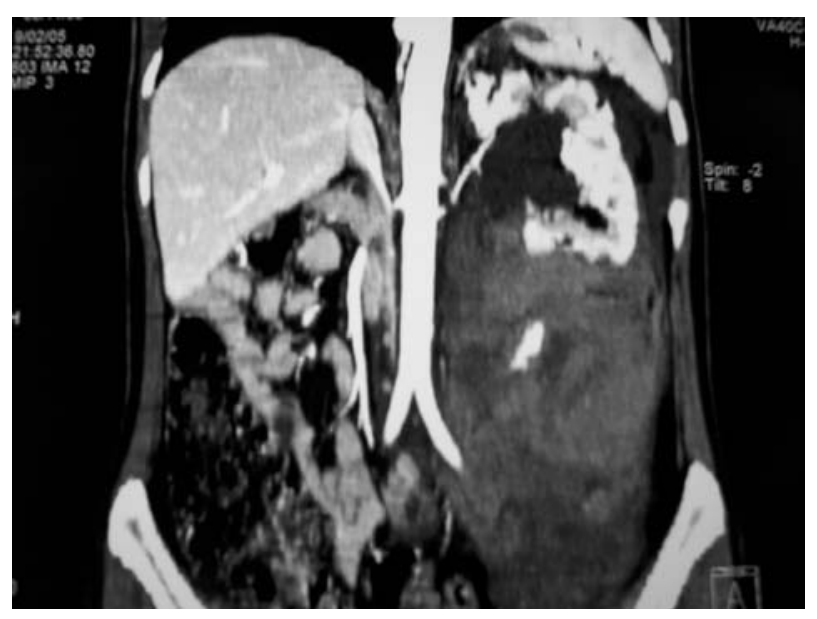

FIGURA 1

Cuando valoramos a la paciente, esta presentaba un abdomen con reacción peritoneal difusa y una masa en flanco izquierdo, junto con claros signos de shock hipovolémico, con palidez mucocutánea y mal relleno capilar. La tensión arterial experimentó una caída brusca, con una tensión sistólica máxima de $70 \mathrm{~mm}$ de $\mathrm{Hg}$ y una frecuencia cardiaca de $70 \mathrm{lpm}$, que no remontaba con infusión de volumen. A esto se sumó una caída de 7 puntos del hematocrito y una creatinina sérica de 2,3, con cifras previas normales, tras haber transcurrido 3,5 horas desde la admisión de la paciente en el Servicio de Urgencias.

Ante la rápida instauración de un cuadro de inestabilidad hemodinámica severa en la paciente, se decide la realización de laparotomía explo- radora y control de la hemorragia con conservación de parénquima si fuera posible, de forma urgente, asumiendo el alto riesgo de pérdida de la unidad renal en esta paciente monorrena.

Durante el acto quirúrgico se accede a la cavidad abdominal a través de una laparotomía media, evacuando un hemoperitoneo en torno a $750 \mathrm{ml}$. La exploración del retroperitoneo revela un gran hematoma que ocupa toda la celda renal, evacuándose al abrir el espacio parietocólico izquierdo. La exploración del riñón pone de manifiesto múltiples AML en toda su superficie, tres de ellos con sangrado activo, que se extirpan, realizando sutura hemostática del lecho con material reabsorbible sobre parches de Surgicel®. No fue necesaria la disección y clampaje temporal del pedículo renal. Tras confirmar la no existencia de ningún otro punto de sangrado activo, se deja un drenaje tipo penrose en la celda renal, se cierra la apertura peritoneal del espacio parietocólico y la laparotomía media. Durante toda la cirugía, el anestesista procedió a la reposición de la volemia mediante concentrados de hematies y expansores de volumen, en total, se transfundieron 6 concentrados.

La anatomía patológica informa de varios fragmentos de tejido de color marrón oscuro y consistencia elástica, que tras el estudio microscópico fueron informados como AML.

El curso postoperatorio fue favorable. Al día siguiente de la cirugía, la paciente tenía una hemoglobina de 7 , precisando la transfusión de 2 concentrados de hematies más. Durante el resto de su estancia hospitalaria, de 7 dias en total, la paciente permaneció con valores estables de hematocrito y se normalizó la función renal, presentando una creatinina de $0^{\circ} 9$ en el momento del alta hospitalaria.

La dilatación de la pelvis renal izquierda informada en el TAC realizado de urgencia fue estudiada en la consulta por medio de urografía intravenosa. En las placas se observaba un riñón izquierdo aumentado de tamaño, con un contorno pobremente definido, en el que destaca una gran dilatación de la pelvis y de los cálices, sin visualizarse el uréter, orientando todo ello a un síndrome de la unión pieloureteral (Fig. 2). Cuando se administra el contraste aparece una vejiga perfectamente contrastada y de características normales. 


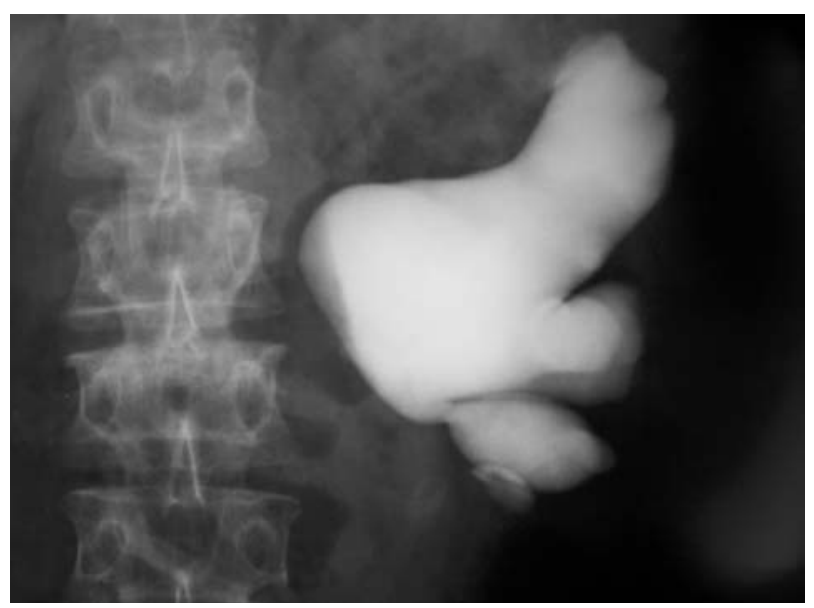

FIGURA 2

La paciente se encontraba en ese momento asintomática y con función renal normal, sin historia de dolor lumbar ni infecciones urinarias. Se decide actitud expectante, con observación y controles seriados por medio de ecografia y urografía intravenosa.

Han pasado 20 meses desde la cirugía practicada de urgencia y la paciente se encuentra asintomática, sin haber presentado nuevos episodios de sangrado retroperitoneal ni clínica correspondiente a su síndrome de la unión pieloureteral.

\section{DISCUSIÓN}

El hematoma perirrenal, subcapsular, intrarrenal o mixto espontáneo o síndrome de Wünderlich consiste en la súbita formación de una colección hemática en la celda renal de etiologia no traumática. Su etiología puede ser diversa. McDougal y col. realizaron una revisión de 78 casos encontrados en la literatura inglesa, reflejando como etiología más frecuente los tumores renales $(57,7 \%)$, seguidos de las patologías vasculares $(17,9 \%)$ y las infecciones (10,3\%). Las discrasias sanguíneas son consideradas por dicho autor debido a su alta frecuencia mientras que el adenocarcinoma renal es una causa rara ${ }^{1}$. Como se ha mencionado, la hemorragia retroperitoneal espontánea puede presentarse como complicación de la terapia anticoagulante. En este contexto se pueden dar dos situaciones muy diferentes, una cuando la hemorragia aparece dentro del rango terapéutico de la anticoagulación, entonces casi siempre hay una causa subyacente de tipo local que lo provoca y entonces hay una indicación quirúrgica, y otra cuando la hemorragia se presenta por haber sobrepasado el rango terapéutico de anticoagulación, en estos casos es excepcional encontrar una causa local que lo provoque y el tratamiento inicial debe ser médico. No obstante, la aparición de complicaciones como IRA, CID, sepsis, signos de compresión nerviosa, pueden hacer variar el criterio a favor de una intervención quirúrgica ${ }^{2}$.

La hemorragia retroperitoneal espontánea puede debutar clínicamente de forma aguda o insidiosa. La presentación típica aunque no patognomónica es la tríada de Lenk que consiste en dolor lumboabdominal agudo, tumoración que ocupa la celda renal y que se extiende al hemiabdomen del mismo lado y deterioro del estado general con signos y síntomas de shock hipovolémico. Cuando la hemorragia es lenta o de escasa cuantía la forma de presentación es insidiosa y progresiva, pudiendo estar estos sintomas atenuados o ausentes.

Entre las pruebas de imagen útiles ante esta situación clínica contamos con la ecografia, técnica no invasiva y que nos sirve como una primera aproximación. Valora el contorno renal y detecta masas perirrenales, aunque no distingue si estas son sólidas o coágulos organizados.

La urografía intravenosa puede mostrar la lesión renal causante del hematoma e informarnos sobre el estado funcional del otro riñón, desviaciones del uréter, extravasación de contraste,...

El TAC es el método de imagen para llegar al diagnóstico $^{3}$ y el más rápido en una situación de inestabilidad hemodinámica. Este procedimiento permite observar una masa perirrenal de moderada atenuación que desplaza y comprime las estructuras retroperitoneales adyacentes, así como determinar la presencia de un tumor o no, y distinguir la existencia de un AML por la densidad grasa. Es muy útil en el seguimiento de los hematomas retroperitoneales tratados de forma conservadora a la vez que contribuye en el diagnóstico diferencial con la rotura de aneurisma de aorta abdominal ${ }^{4}$.

La arteriografía resulta de utilidad en aquellos casos en que no se puede llegar a un diagnóstico mediante TAC (periarteritis, aneurismas, malformaciones arteriovenosas, pequeños hipernefromas o microaneurismas) o bien como método terapéutico en aquellos hematomas retroperitoneales con inestabilidad hemodinámica. Suele aparecer como una masa avascular, sin visualización de la cápsula renal y con desplazamiento de las arterias capsulares respecto a la cápsula y el parénquima renal ${ }^{4}$. 
En el caso que nos ocupa, el causante de la hemorragia es un AML en un riñón único. Se trata de una de las causas más frecuentes de síndrome de Wünderlich sólo precedida por las neoplasias renales malignas. Se trata de un hamartoma, con presencia de haces de células musculares con frecuentes atipias celulares, adipocitos más o menos maduros, y vasos neoformados, en ocasiones de características aneurismáticas, cuya rotura espontánea será la causa del sangrado. Las frecuentes atipias celulares han dado lugar a discusiones con respecto a su capacidad para malignizarse. En cualquier caso, no hay descritas metástasis comprobadas, existiendo casos múltiples y multicéntricos. No obstante, no compete a este artículo la discusión de las formas de presentación, diagnóstico y tratamiento de los angiomiolipomas, ampliamente descritos en la literatura.

El tratamiento de la hemorragia retroperitoneal espontánea es un tema controvertido. Hace años se postulaba la cirugía radical en el caso en que el riñón contralateral fuera sano debido a la alta incidencia de hipernefromas de pequeño tamaño en situación periférica que no se detectan por medio de las técnicas de imagen disponibles ${ }^{5}$. Hoy en día la mayoría de los autores rechazan esta postura y defienden el tratamiento conservador en todos aquellos casos en que se mantenga la estabilidad hemodinámica permitiendo un mejor estudio posterior con una cirugia diferida si es precisa. Existe en la literatura la comunicación de un caso en que se practicó un tratamiento conservador junto con una cirugía laparoscópica diferida para eliminar la causa desencadenante. El autor describe una cirugía más compleja debido a la presencia de fibrosis, aunque no imposible, según la experiencia y habilidad de cirujano ${ }^{6}$.

Por otro lado, en los casos de sangrado retroperitoneal con repercusión hemodinámica se han de tomar las debidas medidas de soporte cardiocirculatorio, asegurando en primer lugar la vía aérea y una correcta oxigenación tisular. Una vez realizado esto, se ha de contar con unos buenos accesos venosos, ya sea con vías venosas periféricas de calibre grueso o centrales. En el caso en que el volumen sanguíneo perdido estimado sea inferior al 20\%, la tensión arterial se podrá sostener con la infusión de sustancias cristaloides o coloideas. En cambio, cuando este sea superior al $30 \%$, será necesario el aporte de derivados sanguíneos. En cuanto al tratamiento etiológico, cuando se sospeche que la causa desencadenante de la hemorragia se trata de una alteración de la coagulación, este ha de hacerse mediante la infusión de plasma y factores de la coagulación. Sin embargo, cuando la causa desencadenante sea una lesión renal, en nuestro caso un angiomiolipoma, existen dos opciones de tratamiento, la arteriografía y embolización selectiva cuando sea posible y la cirugía urgente con extirpación de la lesión sangrante. Es muy importante el realizar una cirugía conservadora de nefronas, sobre todo en los casos en que la causa de la hemorragia sea la ruptura de un AML, ya que estos suelen ser múltiples y precisar de cirugía en algún otro momento. Esta es una de las ventajas de la arteriografía con embolización selectiva.

En el caso de nuestra paciente, quizá parezca más apropiado haber realizado una arteriografía, ya que se trata de una paciente monorrena que perdió la otra unidad renal por una situación clínica similar, pero la rápida inestabilización de la paciente nos decidió por una cirugía urgente, en nuestro medio más rápida que una arteriografía con embolización selectiva, pudiendo conservar la unidad funcional. Por otro lado, el TAC realizado de forma preoperatorio, ya advertía de la presencia de varios angiomiolipomas, y su embolización hubiera sido complicada.

\section{REFERENCIAS}

1. McDougal WS, Kursh ED, Persky L. Spontaneous rupture of the kidney with perirenal hematoma. J Urol. 1975;114(2):181-184.

2. Estivill $\mathrm{J}$ et al. Complicaciones graves del tratamiento anticoagulante: hemorragia retroperitoneal espontánea. An Med Intern. 1984;2:447-453.

3. Belville JS, Morgentaler A, Loughlin KR, Tumeh SS. Spontaneous perinephric and subcapsular hemorrhage: Evaluation with CT US and angiography. Radiology 1989;172(3):733 -738.

4. Chesa Ponce N, Artiles Hernandez JL, Ponce Socorro JM, del Rosario Medina J, Castro Lopez-Torrella V, et al: Sindrome de Wúnderlich como primera manifestación de un angiomiolipoma renal. Arch Esp Urol. 1995;48(3):305-308.

5. Kendall AR, Senay BA, Coll ME: Spontaneous subcapsular renal hematoma: Diagnosis and managment. J Urol. 1988;139(2):246-250.

6. Hernández F, Ong AM, Rha KH, Pinto PA, Kavoussi LR. Laparoscopic renal surgery alter spontaneous retroperitoneal hemorraghe. J Urol. 2003;170(3):749-751.

Correspondencia autor: Dra. C. Aguilera Tubet.

Servicio de Urología. Hospital Univ. Marqués de Valdecilla.

Santander. Avda. Valdecilla $\mathrm{n}^{\circ} 25$,

39008 Santander (Cantabria). Telf. 942315004

E-mail autor: car1677@hotmail.com

Información artículo: Nota clínica

Trabajo recibido: diciembre 2005

Trabajo aceptado: noviembre 2006 